\title{
Fucose Migration in Intact Protonated Glycan Ions: A Universal Phenomenon in Mass Spectrometry
}

\author{
Eike Mucha, Maike Lettow, Mateusz Marianski, Daniel A. Thomas, Weston B. Struwe, \\ David J. Harvey, Gerard Meijer, Peter H. Seeberger, Gert von Helden,* and Kevin Pagel*
}

\begin{abstract}
Fucose is an essential deoxysugar that is found in a wide range of biologically relevant glycans and glycoconjugates. A recurring problem in mass spectrometric analyses of fucosylated glycans is the intramolecular migration of fucose units, which can lead to erroneous sequence assignments. This migration reaction is typically assigned to activation during collision-induced dissociation (CID) in tandem mass spectrometry (MS). In this work, we utilized cold-ion spectroscopy and show for the first time that fucose migration is not limited to fragments obtained in tandem MS and can also be observed in intact glycan ions. This observation suggests a possible lowenergy barrier for this transfer reaction and generalizes fucose migration to an issue that may universally occur in any type of mass spectrometry experiment.
\end{abstract}

Carbohydrates, or glycans, are one of the four most important classes of biomolecules. Their immense structural diversity enables diverse biological functions, such as cell signaling or molecular recognition. ${ }^{[1]}$ However, the complex nature of glycans renders detailed structural analyses difficult. Typically, sophisticated MS-based techniques are used in combination with chromatography ${ }^{[2]}$ or ion-mobility spectrometry. ${ }^{[3]}$ Another powerful tool to investigate molecules in the gas phase is the combination of mass spectrometry (MS) and infrared (IR) spectroscopy. For peptides and proteins, this approach can be used, for example, to deduce information

[*] E. Mucha, M. Lettow, Dr. M. Marianski, Dr. D. A. Thomas, Prof. Dr. G. Meijer, Prof. Dr. G. von Helden, Prof. Dr. K. Pagel Fritz-Haber-Institut der Max-Planck-Gesellschaft

Faradayweg 4-6, 14195 Berlin (Germany)

E-mail: helden@fhi-berlin.mpg.de

E. Mucha, M. Lettow, Prof. Dr. K. Pagel

Institut für Chemie und Biochemie der Freien Universität Berlin Takustraße 3, 14195 Berlin (Germany)

E-mail: kevin.pagel@fu-berlin.de

Prof. Dr. P. H. Seeberger

Max-Planck-Institut für Kolloid- und Grenzflächenforschung

Am Mühlenberg 1, 14476 Potsdam (Germany),

and

Institut für Chemie und Biochemie der Freien Universität Berlin Arnimallee 22, 14195 Berlin (Germany)

Dr. W. B. Struwe

Oxford Glycobiology Institute, Department of Biochemistry University of Oxford, Oxford OX1 3QU (UK)

Dr. D. J. Harvey

Target Discovery Institute, Nuffield Department of Medicine University of Oxford, Roosevelt Drive, Oxford OX3 7FZ (UK)

(-) Supporting information and the ORCID identification number(s) for

(D) the author(s) of this article can be found under:

https://doi.org/10.1002/anie.201801418. about functional groups, secondary structure elements, or preferred molecular conformations. Recent studies show that highly diagnostic IR fingerprints can also be obtained for carbohydrates by using cold-ion spectroscopy. ${ }^{[4]}$ This applies not only to intact glycan ions, but also to their diagnostic fragments, which can be used to deduce structural information. $^{[5]}$

Recently, we investigated isomeric, fucose-containing tetrasaccharides using cold-ion IR spectroscopy and reported their characteristic optical signatures, which allow them to be unambiguously distinguished. ${ }^{[4 a]}$ Fucose is a prevalent monosaccharide building block that is found in N-glycans, Oglycans, and milk-sugars and is typically attached in the final step of glycan processing. A distinct feature of fucose is the lack of a hydroxy group at the C-6 position, which makes it a deoxy sugar (Figure $1 \mathrm{~b}$ ). Biologically relevant examples of

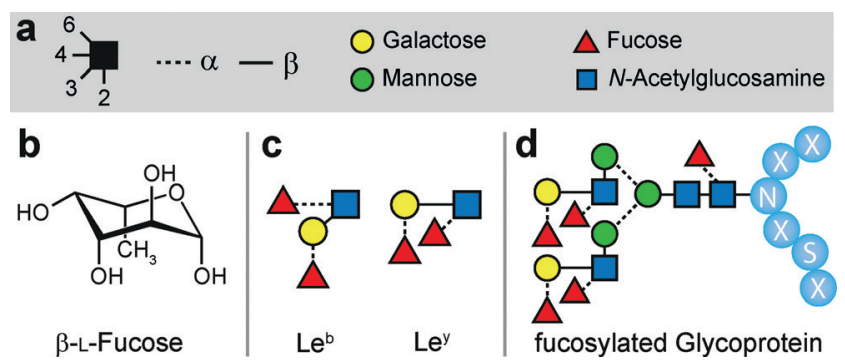

Figure 1. a) Symbol nomenclature for glycans $\left(S N F G^{[8]}\right)$. b) L-Fucose is a ubiquitous monosaccharide building block that lacks a hydroxy group at the C- 6 position. c) Lewis b and Lewis y tetrasaccharides as an example for fucosylated oligosaccharides. d) A highly fucosylated Nglycan extracted from a glycoprotein found in parotid gland tissue. ${ }^{[9]}$

fucose-containing glycans can be found in the $\mathrm{ABO}$ and Lewis blood group system. One example is the Lewis b ( $\mathrm{Le}^{\mathrm{b}}$ ) antigen, a fucosylated tetrasaccharide epitope that is widely distributed throughout the human body. The corresponding isomer Lewis y $\left(\mathrm{Le}^{\mathrm{y}}\right)$, on the other hand, is expressed on a wide range of tumors and can serve as a biomarker ${ }^{[6]}$ (Figure 1c). In general, glycoconjugates such as glycoproteins often exhibit distinct fucosylation patterns that are altered dynamically during the development of cancer. ${ }^{[7]}$

A frequently observed phenomenon for fucose-containing glycans in tandem MS experiments is the intramolecular transfer of terminal fucose units to adjacent or remote monosaccharides upon activation. ${ }^{[10]}$ This so-called fucose migration often results in misleading fragment ions, which in turn can lead to erroneous structural assignments. Here, we focus on a spectroscopic investigation of fucosylated glycans 
and present the first direct evidence for fucose migration in intact protonated glycan ions. Our results show that fucose migration is not only related to tandem MS experiments, but is a genuine issue in all branches of mass spectrometry.

The experimental setup has previously be described in great detail and the interested reader is referred to the scientific literature. ${ }^{[4 a, 11]}$ Briefly, molecular ions are generated by nano-electrospray ionization, mass-to-charge selected in a quadrupole mass filter, and accumulated in a hexapole ion trap. Superfluid helium droplets containing on average $10^{5}$ helium atoms traverse the trap and pick up ions, which are then rapidly cooled to the equilibrium temperature of the droplet $(0.4 \mathrm{~K})$. Downstream, the cryogenic ions inside the helium droplets are investigated using the Fritz Haber Institute IR free-electron laser (FHI-FEL ${ }^{[12]}$ ), and a highly reproducible IR spectrum is recorded. For intact glycan ions, soft source conditions are used to transfer the ions into the mass spectrometer and avoid excessive activation (Figure S1 in the Supporting Information). Fragments are obtained using harsher source conditions to induce dissociation of intact glycan ions (Figure S2).

First, glycans of the $\mathrm{Le}^{\mathrm{y}}$-series were investigated as sodium adducts. Activation of the $\left[\mathrm{Le}^{\mathrm{y}}+\mathrm{Na}\right]^{+}$precursor ions $(\mathrm{m} / \mathrm{z}=$ 698) yields an abundant signal, which corresponds to the neutral loss of a single fucose unit $(\mathrm{m} / \mathrm{z}=552)$. Depending on which of the two fucose residues is lost upon fragmentation, two resulting fragment structures are possible (Figure $2 \mathrm{a}$ ). Because both trisaccharide fragment structures share the same mass, a mass spectrometric analysis alone cannot reveal whether one fragment structure is preferably formed over the other. However, both candidate trisaccharide structures are also available as standards: Lewis $\mathrm{x}\left(\mathrm{Le}^{\mathrm{x}}\right)$ and blood group antigen $\mathrm{H}-2$ (BG-H2). Using the experimental setup described above, IR spectra for the sodium adducts of the $\mathrm{Le}^{\mathrm{y}}$ fragment as well as the trisaccharide standards $\mathrm{Le}^{\mathrm{x}}$ and BG$\mathrm{H} 2$ were recorded (Figure $2 \mathrm{~b}$ ). All three IR spectra show well-resolved absorption features between $1000 \mathrm{~cm}^{-1}$ to $1700 \mathrm{~cm}^{-1}$. Strong absorptions around $1100 \mathrm{~cm}^{-1}$ are usually attributed to $\mathrm{C}-\mathrm{O}$ stretch vibrations, and the features at $1520 \mathrm{~cm}^{-1}$ and $1680 \mathrm{~cm}^{-1}$ likely stem from the characteristic amide II $(\mathrm{N}-\mathrm{H}$ bend $)$ and amide $\mathrm{I}(\mathrm{C}=\mathrm{O}$ stretch $)$ vibrations of the $\mathrm{N}$-acetylglucosamine residue, respectively. Whereas the two trisaccharides $\mathrm{Le}^{\mathrm{x}}$ and $\mathrm{BG}-\mathrm{H} 2$ can be unambiguously distinguished by their unique spectral signatures, the $\operatorname{Le}^{\mathrm{y}}$ fragment spectrum shares absorption features with both trisaccharide spectra. If both trisaccharides are formed during fragmentation, the resulting IR spectrum is expected to be a linear combination of the two individual trisaccharide spectra. And indeed, the linear combination (Figure 2c) matches the fragment spectrum well and suggests an approximate fragment ratio of $1: 1\left(\mathrm{Le}^{\mathrm{x}} / \mathrm{BG}-\mathrm{H} 2\right)$.

Previous studies have utilized ion-mobility mass spectrometry (IM-MS) to study Lewis and blood group antigens and their characteristic fragment ions. For sodium adducts, distinct collision cross sections (CCSs) are obtained for the $\mathrm{Le}^{\mathrm{x}}$ and $\mathrm{BG}-\mathrm{H} 2$ trisaccharides, and both structures can be identified as fragments upon activation of the $\mathrm{Le}^{\mathrm{y}}$ precursor ion. ${ }^{[3 \mathrm{~b}]}$ For the protonated species, however, both trisaccharides and the Le $\mathrm{e}^{\mathrm{y}}$ fragment show identical CCS values (see the
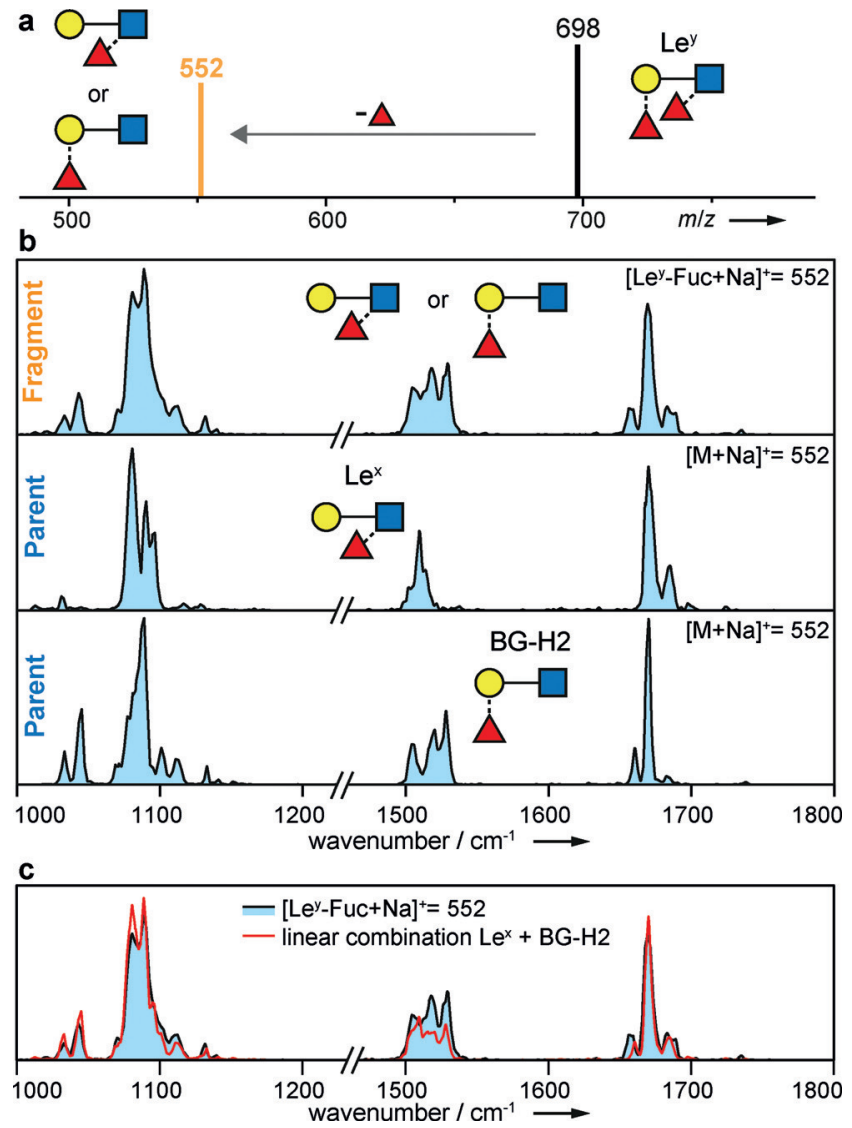

Figure 2. a) Schematic tandem MS spectrum of the $\mathrm{Le}^{\mathrm{y}}$ tetrasaccharide as $[\mathrm{M}+\mathrm{Na}]^{+}$species. The major fragment $(\mathrm{m} / \mathrm{z} 552)$ results from the neutral loss of one fucose unit. A complete mass spectrum is shown in the Supporting Information b) IR spectra of $\left[\mathrm{Le}^{\mathrm{y}} \text {-Fuc }+\mathrm{Na}\right]^{+}$fragment and intact precursor standards $\mathrm{Le}^{\mathrm{x}}$ and $\mathrm{BG}-\mathrm{H} 2 \mathrm{~m} / \mathrm{z} 552$. c) The IR spectrum of the sodiated Le $\mathrm{e}^{\mathrm{y}}$ fragment can be represented by a linear combination of the individual spectra of the trisaccharide standards Le $\mathrm{x}^{\mathrm{x}}$ and $\mathrm{BG}-\mathrm{H} 2$.

Supporting Information). In order to investigate in how far similar CCS values for these protonated glycans can be attributed to similar three-dimensional structures, cold-ion IR spectroscopy was employed.

The protonated $\mathrm{Le}^{\mathrm{y}}$ precursor ions $(m / z=676)$ show a similar fragmentation pattern as the previously described sodium adducts. An abundant signal corresponding to the neutral loss of a single fucose unit $(\mathrm{m} / \mathrm{z}=530)$ is observed. Again, two resulting fragment structures are possible depending on which fucose unit is lost upon fragmentation (Figure $3 \mathrm{a}$ ). The corresponding IR spectrum of this fragment was recorded (Figure $3 \mathrm{~b}$ ) and displays a number of resolved bands between $1000-1200 \mathrm{~cm}^{-1}$ and a single band around $1680 \mathrm{~cm}^{-1}$. Next, the two trisaccharide standards $\mathrm{Le}^{\mathrm{x}}$ and $\mathrm{BG}-\mathrm{H} 2$ were individually investigated since they exhibit the two different structures of the possible fragment ions. Interestingly, the IR spectra of the protonated precursor ions $\mathrm{Le}^{\mathrm{x}}$ and $\mathrm{BG}-\mathrm{H} 2$, as well as the Le ${ }^{\mathrm{y}}$ fragment spectrum, are nearly identical. Every significant absorption band is present in the individual spectra, and only the relative intensity of some bands differs slightly between the $\mathrm{Le}^{\mathrm{y}}$ fragment and the trisaccharide standards. This finding is particularly surprising and contra- 

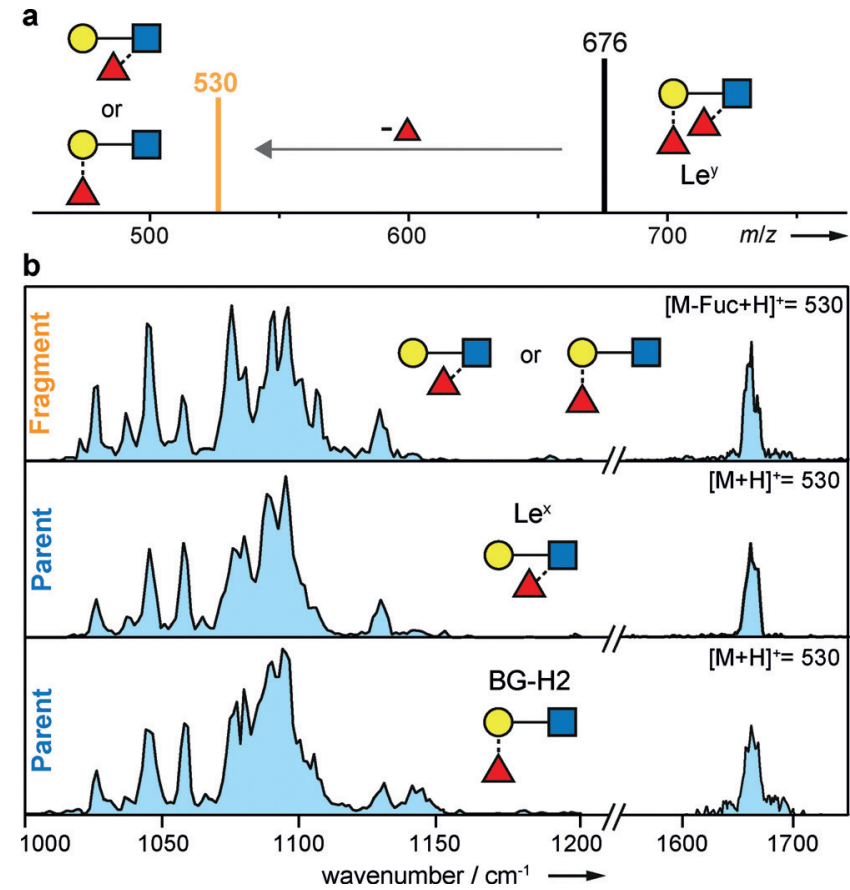

Figure 3. a) Schematic tandem MS spectrum of the Le $\mathrm{e}^{y}$ tetrasaccharide as $[M+H]^{+}$species. The major fragment $(m / z 530)$ results from the neutral loss of one fucose unit. A complete mass spectrum is shown in the Supporting Information b) IR spectra of fragment and intact precursor standards $\mathrm{Le}^{\mathrm{x}}$ and $\mathrm{BG}-\mathrm{H} 2(\mathrm{~m} / \mathrm{z} 530)$.

dictory to previous results, which show that this experimental technique is able to resolve subtle structural variations, even the stereochemical orientation of a single hydroxy group. But why does this highly diagnostic method yield identical IR signatures for these structural isomers?

The likelihood that both trisaccharides coincidentally yield the same IR spectrum is negligibly small, and thus only one plausible explanation remains: the protonated $\mathrm{Le}^{\mathrm{y}}$ fragment, as well as both protonated trisaccharide standards $\mathrm{Le}^{\mathrm{x}}$ and $\mathrm{BG}-\mathrm{H} 2$, undergo a rearrangement reaction, that is, a fucose migration, to the same structure, which leads to three identical IR signatures. A possible explanation for the different absorption intensities of individual bands (such as the band at $1040 \mathrm{~cm}^{-1}$ ) is a different ratio of alpha and beta anomers between the intact ions and the activated fragment. The small absorption band at $1145 \mathrm{~cm}^{-1}$, which is only found in the spectrum of BG-H2, may stem from a small fraction of either a different molecular conformation or a different rearrangement product.

In contrast to previous tandem MS experiments, which indirectly indicate fucose migration by the occurrence of misleading fragment ions, these results present the first direct evidence for this elusive structural rearrangement. It has been proposed $^{[10 \mathrm{a}]}$ that fucose migration is caused by activation during collision-induced dissociation (CID) of precursor ions. Observing this rearrangement in intact ions, however, clearly shows that fragmentation is not a prerequisite for fucose migration. The energy barrier for this reaction can likely not be generalized and strongly depends on the glycan structure. In this work, however, soft source conditions were used to study the intact trisaccharide ions and minimize ion activation during transfer into the mass spectrometer. Therefore, a low activation energy barrier can be expected for this process. The sodium adducts, on the other hand, do not undergo fucose migration and therefore show distinct IR signatures (Figure $2 \mathrm{~b}$ ). This observation is in full agreement with previous reports, which shows that fucose migration can be efficiently inhibited using sodium instead of proton adducts.

Fucose-transfer reactions are generally associated with the presence of mobile protons, but the final structural motif as well as the underlying mechanism remain obscure. In principle, the highly resolved IR spectra obtained in this work can be matched to candidate structures derived from ab initio methods to gain insight into the fucose migration mechanism. However, the large number of possible rearrangement products and the unknown mechanism render this an exceedingly difficult, if not virtually impossible, task.

Interestingly, the related isomeric trisaccharides Lewis a $\left(\mathrm{Le}^{\mathrm{a}}\right)$ and BG-H type 1 (BG-H1) of the Lewis b series exhibit different IR signatures for both the protonated species and sodium adducts (Figures S3 and S4). The distinct IR spectra of protonated $\mathrm{Le}^{\mathrm{a}}$ and $\mathrm{BG}-\mathrm{H} 1$ show some similarity to the singly defucosylated Lewis b fragment. This observation may indicate either that fucose migration is inhibited for those structures or that the products after a possible rearrangement reaction are different.

In summary, cold-ion infrared spectroscopy reveals that fucose migration can occur in intact ions and is not necessarily a result of collision-induced dissociation (CID). Therefore, fucose migration must be regarded as a prevalent issue in the mass spectrometric analysis of protonated glycan ions that may occur in any type of mass spectrometry experiment. The exact mechanism and the final product of the transfer reaction remain elusive, however, it is likely that charge migration catalyzes the rearrangement of individual fucose residues to spatially adjacent sites. The occurrence of fucose migration in intact, protonated precursor ions furthermore indicates a potentially low activation energy barrier. In metal adducts, on the other hand, proton transfer cannot take place, and the localized charge prevents the rearrangement reaction. Further experiments using site-specific isotope labeling of glycans and extensive theoretical calculations are required to fully understand the underlying processes.

\section{Acknowledgements}

The authors gratefully acknowledge generous funding by the Max-Planck-Society and the great expertise of Sandy Gewinner and Dr. Wieland Schöllkopf of the FHI free electron laser. Furthermore, we acknowledge Dr. Fabian Pfrengle for useful and thorough discussions. D.A.T acknowledges support from the Alexander von Humboldt Foundation.

\section{Conflict of interest}

The authors declare no conflict of interest. 
Keywords: carbohydrates - fucose - glycans - IR spectroscopy mass spectrometry

How to cite: Angew. Chem. Int. Ed. 2018, 57, 7440-7443 Angew. Chem. 2018, 130, 7562-7565

[1] a) A. Varki, Glycobiology 1993, 3, 97-130; b) R. A. Dwek, Chem. Rev. 1996, 96, 683-720.

[2] a) K. Mariño, J. Bones, J. J. Kattla, P. M. Rudd, Nat. Chem. Biol. 2010, 6, 713; b) L. Veillon, Y. Huang, W. Peng, X. Dong, B. G. Cho, Y. Mechref, Electrophoresis 2017, 38, 2100-2114; c) N Viseux, E. de Hoffmann, B. Domon, Anal. Chem. 1997, 69 3193-3198; d) L. R. Ruhaak, A. M. Deelder, M. Wuhrer, Anal. Bioanal. Chem. 2009, 394, 163-174.

[3] a) J. Hofmann, H. S. Hahm, P. H. Seeberger, K. Pagel, Nature 2015, 526, 241-244; b) J. Hofmann, A. Stuckmann, M. Crispin, D. J. Harvey, K. Pagel, W. B. Struwe, Anal. Chem. 2017, 89 $2318-2325$; c) P. Both, A. P. Green, C. J. Gray, R. Sardzik, J. Voglmeir, C. Fontana, M. Austeri, M. Rejzek, D. Richardson, R. A. Field, G. Widmalm, S. L. Flitsch, C. E. Eyers, Nat. Chem. 2014, 6, 65-74; d) H. Hinneburg, J. Hofmann, W. B. Struwe, A. Thader, F. Altmann, D. Varon Silva, P. H. Seeberger, K. Pagel, D. Kolarich, Chem. Commun. 2016, 52, 4381 - 4384.

[4] a) E. Mucha, A. I. González Flórez, M. Marianski, D. A. Thomas, W. Hoffmann, W. B. Struwe, H. S. Hahm, S. Gewinner, W. Schollkopf, P. H. Seeberger, G. von Helden, K. Pagel, Angew. Chem. Int. Ed. 2017, 56, 11248-11251; Angew. Chem. 2017, 129 11400-11404; b) C. Masellis, N. Khanal, M. Z. Kamrath, D. E. Clemmer, T. R. Rizzo, J. Am. Soc. Mass Spectrom. 2017, 28, 2217-2222.

[5] B. Schindler, L. Barnes, G. Renois, C. Gray, S. Chambert, S. Fort, S. Flitsch, C. Loison, A.-R. Allouche, I. Compagnon, Nat. Commun. 2017, 8, 973.

[6] a) B. W. T. Yin, C. L. Finstad, K. Kitamura, M. G. Federici, M. Welshinger, V. Kudryashov, W. J. Hoskins, S. Welt, K. O. Lloyd, Int. J. Cancer 1996, 65, 406-412; b) X. Xie, M. Boysen, O. P. F.
Clausen, M. A. Bryne, Laryngoscope 1999, 109, 1474-1480; c) R. B. Myers, S. Srivastava, W. E. Grizzle, J. Urol. 1995, 153, $1572-1574$.

[7] J. Le Pendu, S. Marionneau, A. Cailleau-Thomas, J. Rocher, B. Le Moullac-Vaidye, M. Clément, APMIS 2001, 109, 9-26.

[8] A. Varki, R. D. Cummings, M. Aebi, N. H. Packer, P. H. Seeberger, J. D. Esko, P. Stanley, G. Hart, A. Darvill, T. Kinoshita, J. J. Prestegard, R. L. Schnaar, H. H. Freeze, J. D. Marth, C. R. Bertozzi, M. E. Etzler, M. Frank, J. F. Vliegenthart, T. Lutteke, S. Perez, E. Bolton, P. Rudd, J. Paulson, M. Kanehisa, P. Toukach, K. F. Aoki-Kinoshita, A. Dell, H. Narimatsu, W. York, N. Taniguchi, S. Kornfeld, Glycobiology 2015, 25, 1323 1324.

[9] G. R. Guile, D. J. Harvey, N. O’Donnell, A. K. Powell, A. P. Hunter, S. Zamze, D. L. Fernandes, R. A. Dwek, D. R. Wing, Eur. J. Biochem. 1998, 258, 623-656.

[10] a) D. J. Harvey, T. S. Mattu, M. R. Wormald, L. Royle, R. A. Dwek, P. M. Rudd, Anal. Chem. 2002, 74, 734-740; b) A. H. Franz, C. B. Lebrilla, J. Am. Soc. Mass Spectrom. 2002, 13, $325-$ 337; c) M. Wuhrer, C. A. M. Koeleman, C. H. Hokke, A. M. Deelder, Rapid Commun. Mass Spectrom. 2006, 20, 1747-1754; d) C. Nwosu, H. K. Yau, S. Becht, Anal. Chem. 2015, 87, 59055913.

[11] a) F. Filsinger, D. S. Ahn, G. Meijer, G. von Helden, Phys. Chem. Chem. Phys. 2012, 14, 13370-13377; b) A. I. González Flórez, E. Mucha, D. S. Ahn, S. Gewinner, W. Schöllkopf, K. Pagel, G. von Helden, Angew. Chem. Int. Ed. 2016, 55, 3295-3299; Angew. Chem. 2016, 128, 3356-3360.

[12] W. Schöllkopf, S. Gewinner, H. Junkes, A. Paarmann, G. von Helden, H. Bluem, A. M. M. Todd, Proc. SPIE-Int. Soc. Opt. Eng. 2015, 9512, 95121L.

Manuscript received: February 2, 2018

Revised manuscript received: March 23, 2018

Accepted manuscript online: April 24, 2018

Version of record online: May 25, 2018 